Research Article in Technology

\title{
The Secrets of Consciousness and AI
}

Jaden Q. Zhang ${ }^{1}$

${ }^{1}$ Affiliation 1; email@emailserver.com

* Sacred Heart High School, Ottawa, Ontario K2S 1X4, Canada

(Received: 03/07/2020; Accepted: 04/08/2020; Published: 04/15/2020)

DOI: https://doi.org/10.37906/isteamc.2020.3

\begin{abstract}
Many generations of scientists have tried to understand the concept of consciousness and all its secrets. We can't really define which part of our brain really controls consciousness so it is a topic that most people can't wrap their heads around. The simplest definition I can give to this topic is "when a being is aware of their existence and can feel, they are conscious". I create a situation where we replicate the human brain using computer components to try and isolate or even create consciousness. The closest we have come to really creating a conscience is AI. If we can make our neurons into millions of wires and use many interesting software to replicate our memory would we have a truly self-aware being? Discovering all the secrets of consciousness would really benefit our world in nanotech, and solving world problems from another perspective. I bring together the idea of technology and neuroscience to create and discover new information about AI and how human consciousness works. The idea of transferring human consciousness into a robot would be a new upgraded AI that would solve problems like aging. We would be able to transfer our consciousness only when we can isolate and understand the source of consciousness.
\end{abstract}

Keywords: Consciousness, Artificial Intelligence, Conscious, Neuroscience

\section{Introduction}

What makes you, truly you? Some may say that it's their talents or skills. Some may say it's their physical bodies. The ones who think out of the box say it's what makes them aware and conscious. But let me ask you, what is consciousness? What separates your consciousness from mine? What lets you be you, and me be me? Is it our behaviors or thoughts? When you dive deep into what consciousness is, you will come to a sort of paradox. There is no part of the brain that controls the real you, your brain is split into many parts. To define consciousness is hard, and to prove that something is conscious is harder than you think. Nobody truly knows everything about consciousness and it is still one of the biggest mysteries. In this paper, I want to shine more light on why we need to do more research and show what my perspective on consciousness is. If we understand conscience then our progress in life would propel even faster because of all the technological advancements we can take. The biggest question I want to answer is "What is consciousness?" The research on this subject is not extremely deep; Annaka and numerous other scientists have found out that consciousness is not just tailored to your physical body. The main requirements of consciousness are to have logic and mostly awareness (Harris, 2019). The way to be conscious is to realize and have an imagination, to be able to know and theorize. To the answer the question, I need visit many other subtopics and questions too. I have compiled research from many sources, and I want to bring it all together and hypothesize my own experiment to sum things up. 
Many people have tried to tackle this question many different ways. Scientists, psychologists, and even neurologists have difficulty finding the answers. Some people took the poetic and philosophical path "you are what you feel and what you experience". Some people took a psychological turn on consciousness, and there was also a neuroscientific root. To answer the big question, I have to ask about consciousness in other fields of studies. I would have to use neuroscience and the fact that your brain is just neurons and electric charges to come to a conclusion. Due to the extreme similarity, I can compare mechanical and biological consciousness to see how robots can someday become artificially intelligent (AI), perhaps even have our brains uploaded (Macdonald, 2018). Some people argue that AI solely means that if the robot can trick someone into believing it's a human then it is AI. What my definition of AI is, when we can create a functioning and unique brain where the computer has control and can make its own decisions. If the robot is aware and knowing of its existence in this world, then I consider that true consciousness. I would also move on to the neurological side and say that if you can have multiple memories or experiences and connect them together to make a fictional memory, then that is consciousness or AI. Not only should it be able to have an experience, but it should be able to understand that the experience and everything in it is from this earth. For example, to be conscious you should be able to have neurons fire and take specific models and experiences of objects to decide what a cat is or what a cat is not (Falk, 2019). Many scientists have discovered what consciousness isn't linked to. They know that it is not linked to the body or a separate part of the brain, instead it is the brain as a whole (Harris, 2019). Evolution also has a big part; many centuries of evolution has made the venus flytrap have a memory of sorts. When a gust of wind or rain touches the flytrap, it does not normally close; a fly has to touch two hairs on the plant to register it to close. Therefore, the plant has to remember or sense that the first hair has been touched. However, scientists have deduced that plants are not conscious. The only way plants are conscious is if they have some sort of inner life that philosophers are searching for. The likelihood of that is almost 0 , therefore using probability I can deduce with complete certainty that plants don't have a conscience (Harris, 2019). To add on, most of the time consciousness is just an extra trait to evolution, so only organisms that need and benefit from being conscious will evolve to gain it. Anything mobile should probably have consciousness so that it may hunt and know what it is looking for (Graziano, 2016).

Our brains have to have the capacity of holding an inner world in our heads. We know that something exists even without seeing it. We can understand that dimensions exist and that we are aware of other beings and organisms (Graziano, 2016). I want you to close your eyes. Picture a tree with seven apples on it. Have you ever seen a tree like the one you pictured ever before? If not then that just goes to show how much your conscience works to create models of trees and apples. Without directly looking at one, you can remember and imagine what an apple looks like because your conscience has created a model in your brain (Graziano, 2016).

Most people look at the problem of consciousness, and it really confuses them, because we are thinking about thinking. To wrap our heads around this concept is hard. The only thing we can say for certain is that we humans are conscious, because we created the term, and we set the guidelines of what is conscious and what isn't. Speaking of those boundaries, most people don't know what is considered to be conscious, so in the following, I will dive deep into the requirements for our concept of awareness. I will first be giving a concrete definition of what consciousness is. There are many perspectives to consciousness, so I will visit each idea from different departments, and find one definition that most people would agree upon. There have been many things done with consciousness linking to AI. I will dive deeper into the realm of automatons and the conscience of robots. Since we cannot experiment with consciousness, we need to experiment with AI. The stages of answering my question requires many more questions, like what is $\mathrm{AI}$, or where is consciousness located? We see what has been done already and what scientists hope to do in the future. Then I give a proposal about creating truly conscious AI, and how I would go upon trying that. There would be many obstacles for my idea, but I talk about those topics and find innovative ideas. 
Continuing on the path of $\mathrm{AI}$, an idea is brought to mind about brain scanning. The question of what is consciousness can be answered through scanning our brain and creating technology to replicate what we call a conscience. It is like performing a simulation to find out the answer. We create AI to find the answer to consciousness. With all those ideas in mind, the future would be impacted greatly if we can truly answer the question of "what is consciousness?" Many new discoveries are just on the verge and hopefully this paper can open many doors for people.

\section{Brief Definition}

To really dive into this subject, I need to first find a definition that can fit the word consciousness. There are many people who give it different definitions from their perspectives. There are neurologists, psychologists, and even philosophers who have tried to define human awareness, but I plan to find a main definition that all people agree upon. I found Thomas Nagel's definition to be vague but also true. Thomas Nagel is an American philosopher that went out to find his own definition of consciousness. He claimed that "an organism being conscious is if there is something to be like that organism or the most basic form of experience" (Harris, 2019). When you are conscious, you know you exist and you feel different needs. For example, famish or thirst, those are some things that most conscious beings feel. Many people have found that consciousness takes specific traits. The most basic type of awareness is to feel something as that organism. For example, you can feel tired while a rock cannot, and a ferret can feel famished while a piece of bread cannot. Those kinds of very basic feelings are the bottom of all awareness, meaning if an organism can feel these needs then it is most certainly conscious. If you are not in control of your thoughts and body, then you can't be conscious because you are technically just a robot being programmed. The more conscious you are, the more you're aware of your surroundings. If you are asleep, you are less conscious than when you are awake (Harris, 2019).

Another part of consciousness is the brain. Most people automatically think that the brain controls our conscious thinking. The real question is: what part controls that? The medulla controls breathing, the temporal lobe controls memory and language, and your frontal lobe controls your personality and behavior, but which part is you? The answer to that question is that you are defined by every part of your brain, which in some sense means your consciousness stems from your brain. You cannot just take a single part and call that you. The person that inhabits the body is the entire brain connected together and all working in unison to do one task, that one goal which is to live and reproduce (Hines, 2018). Now that it is established that the whole brain is what a human being's conscience is, we can find out the secrets behind the requirements and how something can become conscious. If you are a lump of matter on the ground, and you suddenly grow a pumping heart, you can say that you are not conscious. If that lump were to grow a brain, you can say it is conscious, but it wouldn't live if it couldn't get an energy source because it has no senses. The lines to conscience and lump are still very fuzzy, but I have compiled some more research, and I would like to present the most basic requirements of consciousness, referencing many neurologists, psychologists and philosophers (Harris, 2019).

On the track of "what is consciousness?" we know where it comes from, so now we need to know when it came to be. Scientists say that conscience arrived from evolution and the survival of not only the fittest but the most aware (Glasgow, 2018). When you think of our past, it is extremely hard to think of a time when we didn't exist, before anything with consciousness existed. Michael Graziano, a psychologist and neurologist, explains how consciousness is one of the most important biological traits any organism can receive. He also explains that we are an evolutionary miracle, our neurons fight over what to do based on what happens. The "loudest most heard" neurons get picked to carry out their decision and actions (Glasgow, 2018). When selective evolution was happening, animals that were "better safe than sorry" were more prone to live and reproduce, therefore giving offspring to more aware animals. Consciousness did not just suddenly appear but happened through multiple stages. When a mammal misinterprets rustling 
grass as a lion that is fine, but if an animal fails to hear the lion, then it is taken out of the gene pool and killed (Graziano, 2016). The original function of awareness was probably to drive something to go and find food (Mashour, 2013). To be conscious, you should also have an image of the world. For example, you should be able to tell the difference between your food and what wants you as food. You should also probably have a sense of space and area. When animals go around hunting, they can visualize the area that they themselves and their food encompasses, which allows the animal to survive and reproduce (Keefe, 2014).

The more senses you have, the better and more aware you are. You can't be a non-moving, non-oral or visual species, because what would you do if that was the case? You can't reproduce and have more conscious lumps around the world. To know what you need to hunt or eat, you need to either be able to see, smell or hear. Those help you in life to find food and a mate. So a part of the definition to be conscious is that you must have some aspect of sense.

\section{Different Perspectives}

There are many perspectives in the field of consciousness. The main ones I want to also cover other than technological and neuroscience are philosophy, psychology, ecology, and what all of the fields have in common. Kristian Marlow, a psychologist, explains how consciousness is extremely hard to explain due to all of the types of consciousness in his field. He talks about the debate that has been going on. He also goes on to explain that consciousness has multiple stages, for example physical and mental connections between neurons. Throughout our life, we build connections between neurons so they can work faster. There are physical connections between them, and ones that work together but from a distance. He uses this idea to construct bridges of the connections in our brain, whether physical or mental (Marlow, 2013).

If I look into ecology or plants and if they are conscious, that is a different level. Earlier I said plants are not conscious, now with a definition and a new perspective we can say otherwise. Annaka Harris explains the processes of memory and understanding in a plant even though it does not have a brain. For example, a venus flytrap has a sort of memory, which ecologists have determined through their experiments. When a single hair on the plant is touched, the plant knows to not close the mouth, because the disturbance is minor, and it could possibly just be some wind. But when two hairs are touched or a considerable force is applied, the mouth closes. Ecologists have determined that the plant has to know, feel, or remember the forces it experiences. There are species of plants that communicate their needs, and also organized attacks on enemy species (Harris, 2019). With that kind of power, it is hard not to see why plants can't also have a form of consciousness. Since they can communicate, organize and even remember (Harris, 2019). It is just not certain yet. There is much more to discover.

In a spiritual manner, consciousness is also related or known as the soul of the person. You can ask the question "at what point are you, not you?" They say if you stripped everything, the only thing you need to be "you" is your soul. In fantasy, a soul is normally portrayed as a little ball of fire in the center of your body or a ghostly spirit. That little ball of fire also represents all your memories and the connections in your brain to make up who you are. Sometimes, objects will have a soul but certainly they don't have a conscience. For example, some may suggest some dolls have souls, but for sure we can determine it does not have awareness (Depung, 2017). To some extent, all these perspectives are connected, and all go off the same root which is Thomas Nagel's definition. They aren't completely different, and we need all perspectives to come up with a final product of consciousness. Also, we need one concrete root of a definition so we can the question. 


\section{Previous Work on AI}

$\mathrm{AI}$ is one of the avenues that we can use to understand conscience. Sometimes learning the subject isn't enough; you have mastered a subject when you can teach and create it. Therefore, I want to reach further into artificial intelligence, so I can wrap all the ideas together and bring this paper to a full circle.

Kevin Schawinski, a well-known astrophysicist, has used AI to do generative modeling and many other things. He does it to try and understand this subject even more. I want to gather some information to give a background on this subject and what everyone else has found. The definition of $\mathrm{AI}$ is a field of engineering and science where a robot can replicate the answers of a human, and trick a person into believing the robot is a human. One of the subfields is speech recognition and image recognition. Humans can tell if something is a cat or not, but robots can't, even with a reference. The computer doesn't truly understand what a cat is, and it won't be able to tell a truck and cat apart. Many corporations have already made many face recognition software to help their program outshine the rest. Many of them are not perfect. Sometimes they can confuse objects that look like faces as actual human faces. The reason they confuse things with each other is because they are not conscious. They may seem conscious on the outside but that doesn't mean they are. It's a state called pseudo awareness (false sense of awareness). The fact that the AI right now is not conscious will help us narrow down the truth of awareness and answer the question. People use things like rules and traits to make the robot as precise as possible. For example, if there is a dark pixel surrounded by light pixels that is a hole or an eye. That program can also count as AI, because it is something that can determine the grouping of an object (Falk, 2019). But AI does not directly correlate to conscience. This is why I want to look into this further, to figure out the differences between AI and consciousness, and maybe how we can create robots that are aware.

There is also another sort of AI which is human conversation. If the program can speak to a person and communicate its own ideas and thoughts, then the program is very much AI. If the programmer didn't just implement a group of answers it can use, then the program is a success. One way they can achieve that is by giving it a sample of answers to different questions, and the program can find the similarities of answers so it can create its own. In the end, you should have a piece of technology that has some form of shallow consciousness, and it should understand the questions and everything. After a while, it can also create its own questions and evolve to making statements. Everything evolves and at a point, it should be independent and not only faking consciousness, but truly obtaining it. Then again to wrap it back around, a shallow form of consciousness is not what I am searching for. If your robot can answer questions with answers, you've never seen before we can call that AI but not awareness, since it doesn't realize its world and the truth of where it resides (Graziano, 2016).

What we need to achieve next to find the answer is uploading our brains to computers as a form of giving a piece of technology consciousness. If we could map our brains and directly put it onto a computer, then that makes our brains immortal, or at least very resilient. Cancers would just turn into bugs and viruses. We would remember more and think faster. We would be super humans. Our brains remember things because our neurons create a passcode of signals when a memory is created. When we remember that memory the passcode is replayed. One of the bigger questions is if all the memories would be kept if only the physical tissue was copied, or would the electrical signals it sent be remembered? What I theorize is that if the brain (on the computer) can get an energy source, then the neurons will fire like they did before. After years of firing a certain way, they adapted to that one certain way. So any energy input will result in the memories being kept and remembered. The future of the brain would change though; it would be able to have storage added to it, and the speed at which it could learn would also increase. This adds to our idea of awareness. We can determine that without a memory, conscience wouldn't happen. Next part, I want to give a proposal which will open up many doors from AI to consciousness. It will answer the question by 
checking the results with the perspectives of psychologists like Kristian Marlow and other professionals that defined awareness (Marlow, 2013).

\section{Proposal}

The question of "what is consciousness?" can be answered by asking more questions. I will go deep into AI to experiment if our brains are the true source of consciousness or not. So the question to ask is, can robots be conscious? Or can we upload our brains to computers? Since I explained what has already been done about AI you will be able to see why this idea is new and very possible. My proposal will be able to answer many different questions, because I already have a concrete definition from Thomas Nagel that I can reference off of, and it can pinpoint exactly the root of our awareness. Many people have created AI, but the definition of it is "a robot that can act like a human or trick a human to think it is conscious even though it may not be human." Many people say that if a robot can trick someone into thinking it is a human, then it is AI. But what I will be referring to in this paper is if a robot is following the definition "an organism being conscious is if there is something to be like that organism or the most basic form of experience." then it is AI. When you search online for robots that can talk to you, they are all programmed to do what they do, but if a robot can create its own decisions, thoughts and know that it exists in this world, then in my case it will be classified as AI. Sophia (as is shown in Figure 1), an android, can create its own decisions and thoughts, yet we can determine it is not conscious by looking at the programming. The lines become very fuzzy because we may not be able to know for sure if the robot understands their existence.

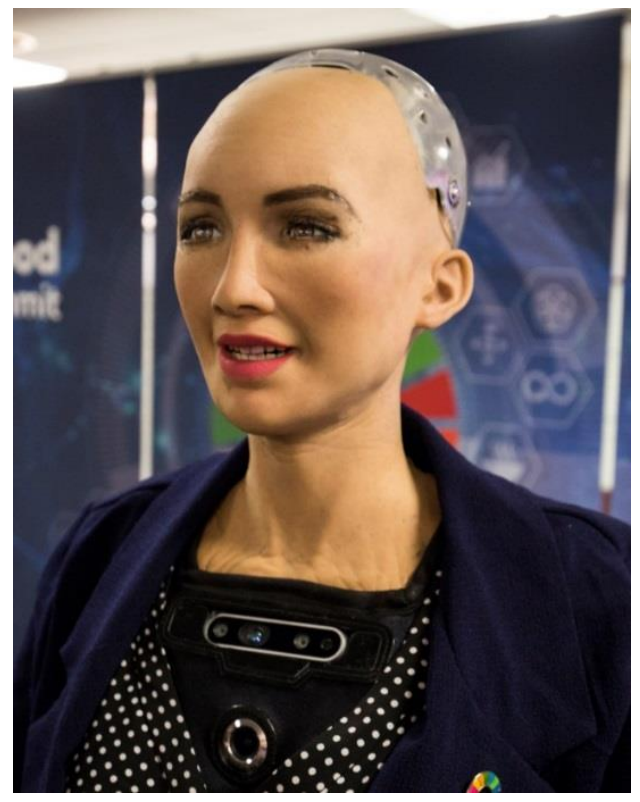

Figure 1. Sophia, a modern AI android

So to unlock the truth on awareness, let me propose an idea on AI. If my brain is made from neurons that fire electricity, then let them be wires. If my thoughts and ideas are a specific firing of those neurons, then let my thoughts be a memory disk. For my logical side of my brain let that be a neural network that learns right from wrong and comparing values. For my creative hemisphere let that be a neural network that learns from different creative answers and learns to create its own ideas.

To be more descriptive on those parts of our brain, let me elaborate. The first part is the neurons. An average human brain has around 100 billion neurons (Cherry, 2019). Many of those neurons develop throughout the human brain, and connect with each other to create memories and different pathways. Every person's brain grows differently due to their own experience, throughout a person's life, their brain 
will make more connections to remember more information. Many people think that people grow more neurons, but that is false. Adults simply have more connections between neurons as they grow, but they have just as many neurons as a developed teenager (Boldrini, 2018). So wires would have to be able to be very thin and extremely plentiful. The next part of the brain that could be recreated would be the thoughts and memories. In the organic human brain, the thoughts and memories are made from specific timings that the neurons fire off signals. To recreate that, you should have a motherboard that is connected to all the wires, and this motherboard extremely complicated. It would be coded with different slots of memory where when specific neurons fire at a specific time, a memory is triggered and brought to a specific area where the brain replays the memory. At the beginning there are no memories, the robot has to create thoughts, and each thought is assigned a group of neurons that fire in an order. After every single possible neuron and order is assigned to a memory, its brain is full. Somehow it needs to have the ability to "forget memories," or we can add an extra memory card to increase the memory capacity. All of that seems pretty extreme, but I'm sure that in a few years, we will have the technology to make that happen. Now that we have found a good substitute for each part of the brain, we can inch closer to finding what consciousness is or how it can be created.

The next two areas of the brain are extremely similar, the sides that tell right from wrong or a dog compared to a cat. They are the two hemispheres of the brain, logical and creative or language. There are two ways to create this. The first way is pretty farfetched, but logically it should function, by using the memory card and fixating on what memory is good and what memory is bad. Just like a human, after many years of telling what is right and what is wrong, it should be able to predict what is right or wrong and react accordingly. Another method would be to use neural networks. A neural network is a program that we feed information into, and tell which information is good and which information is bad. After thousands of examples, the neural network should have created a pattern to determine right from wrong. For the logical hemisphere, I would use a neural network that gave millions of answers right from wrong to let it understand about justice and equality. The creative side would also be a neural network that learns different things like language and emotion from pictures and audio. Then there would be a voice box that takes clips of those voices and merges them together to answer questions. You can tell now that this is true AI consciousness, it's existing and feeling, and we can follow perfectly with Thomas Nagel's definition and they match. For the neural network in language, the original audio would have a question and answers from many people. The robot would learn how to answer that question from the samples, then there would be hundreds of questions with over millions of answers to help the robot. There would also have to be microphones that listen to the questions and match keywords to the sample questions. If you think about it, our consciousness also starts like this; after years of living and experience you learn more. After years of the robot listening and answering questions, it would answer more correctly. Finally, for the robot to depict objects, it would need cameras that can send the video to the motherboard, and it would use the motherboard to try and identify the object, then act accordingly. It would need a source of energy, and for that it could be an outlet for all that matters, but all it needs is energy. With this proposal, the definition and this robot matches perfectly; it can feel emotions and it can feel tired from running out of batteries. This proposal truly answers more questions and we get a better picture of what awareness is. It also helps us pinpoint exact locations of consciousness.

Obviously, there is so much more to a brain then those parts, but imagine we make it possible to replicate each part of the brain with a machine, then we should theoretically be able to have a functioning artificial intelligence. If we give a learning program millions of scans of different kinds of brains and we let it create its own unique brain, by meshing different people's brains together. We should, in theory, have a brain that is unique to itself. We then take the new brain that the machine made and create it using machine parts like I mentioned before and create artificial consciousness. Basically we have a software that takes brain scans and creates a blueprint for a new brain. We then take the blueprint and using my idea of wires 
to neurons etc. We can create a physical working mechanical brain. The brain is just one huge neural network slowly building itself throughout your life, and technically our brain is just an extremely complex machine. But if our machine brain doesn't have a conscience, then we can deduce the brain has nothing to do with consciousness. If we find out that replicating our brain perfectly still doesn't give us a working awareness, then that still answers a part of the question "what is consciousness?" because we can now say that it is not the brain. But that doesn't mean that the AI cannot become conscious after creating a brain of its own. At the moment, our brains are too hard to duplicate intentionally, but since our brain only takes up a finite amount of space, then our cerebrals should theoretically be able to be mapped out. In addition, when humans are pregnant, our body needs to create a brain on its own, which is amazing. As soon as one artificial intelligence creates a functioning brain, then that means they have completed the requirements of conscience to our standards. This area has explained many things and we can take a lot of valuable information out of it. Since consciousness and AI are getting really close to becoming one, as soon as we can create consciousness, we answer the question of this paper.

When I explained that each part of the brain can be created by mechanical parts I know it is possible. Our brain is made through centuries of evolution, and that means someday technology would be advanced enough to replicate the human brain. Our cerebrum is technically a neural network that went through many years of learning. Until that day comes, we should be able to map the human brain on a computer in the near future. Our progress is truly growing at a fast pace, and an article states that our progress is exponential, at the point we are at right now, humans can get to advanced brain mapping in 100-500 years (Dror, 2015). In Crossing Zebra's graph of exponential human innovation, as is shown in Figure 2, you can see that each point is more advanced than the next. Each invention is also closer in time to the next.

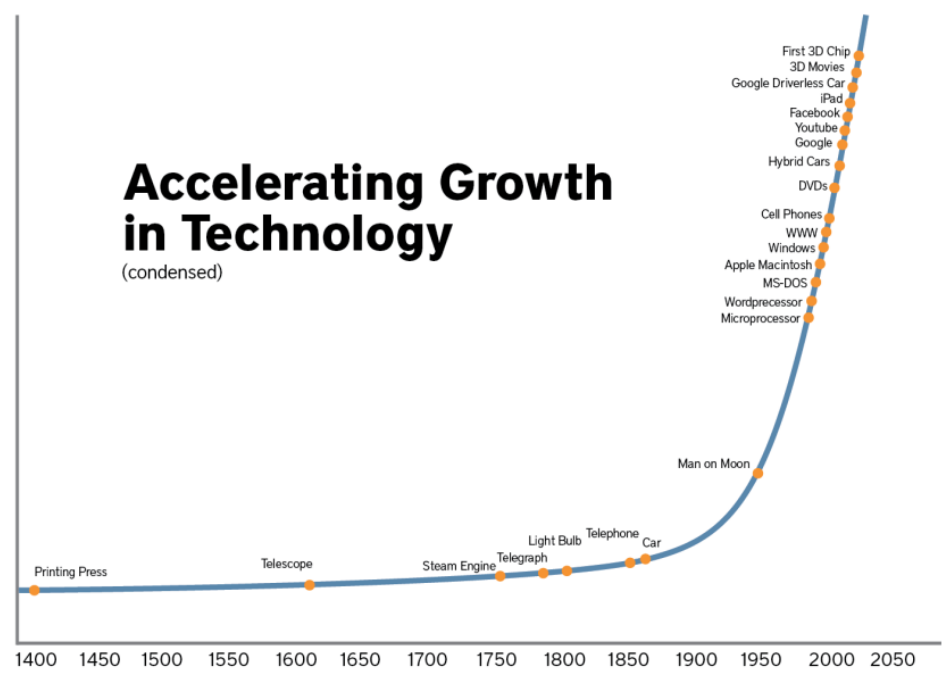

Figure 2. Crossing Zebras graph of exponential human innovation

Many universities and major countries are working on creating a connectome, which is a map where it shows where each part of your brain connects to. Humans have been able to map out only one species brain so far, the c elegans. The difference is that they have only 302 neurons while humans have over 100 billion (Cherry, 2019). The problems I see in my proposal are that this brain cannot be fully compared to a human's brain. The truth is that humans are biological, and over the course of time our brain makes new connections, while computers and AI cannot do that without being organic and growing. When it comes to these sorts of guesses, it's hard to imagine AI becoming organic. If it does, is it even considered a robot anymore? As you can see, my proposal has a few holes in it, but technically if we create a unique brain 
and let it develop, we should have functioning consciousness. The uses of this would be infinite, as we would be able to understand the human brain a lot better and get to unlock many more secrets. For example, we could find how and why illnesses related to the brain occur and either prevent or cure them. That one opportunity would save many thousands of lives. If robots can be conscious with only a brain, then we have answered our question. Consciousness originates from the brain with certainty. In this case, AI directly relates to awareness and our questions. We cannot experiment directly on consciousness, since we do not know what it truly is, but we can experiment on AI which will lead us to the answer.

The proposal I just made is completely possible, but will definitely take very long to achieve. If our brain can be made from evolution, then there must be technology that can replicate each part of the brain. Hopefully in the future, humans can adventure further without having to work and spend energy on other tasks. Many scientists have dedicated their lives to finding the secret about consciousness, but before we can find out the truth we need to see if the brain has anything to do with it at all. There could be some second entity that is like a "soul," and that soul is the real "you". Many types of questions can be answered through this one experiment. There are many things that helped humans take the next step in society, and I think one of those steps needs to be that we should be able to fully understand humans and their motivations. The true answer from testing and experimenting AI then having it match up with all the definitions and opinions of professionals shows that AI can be conscious if it feels emotions or needs. That means that our brain is consciousness as a whole, and that we need to have all parts working together to understand our world. We can only answer our questions if the project is completed, write now we can only imagine the possibilities. I the outcome of the project creates consciousness then we know consciousness relates to the brain. We need to know more about the brain to answer the depths of my questions. We don't understand ourselves or brains completely yet, and I think that is the next leap in our species. Therefore, to understand ourselves more, we have to actually scan our brains. Technology is developing fast; more and more detail is being captured from our brains onto computers. I will be giving more explanations on brain mapping.

\section{Brain Scans and Maps}

Other than giving a new conscience to robots, our brains can be transferred to a robot. To successfully achieve this, we need to fully understand our brains and be able to map them. It is extremely hard to map the brain, because the way everything entangles. People can't even map out one tenth of a millimeter worth of brain matter in a rodent. But since our brains take up a finite amount of space, we will be able to map it out in the future (Cherry, 2019).

The brain will be transferred to a program. A problem we will certainly face is storage space, as brains are extremely powerful and they take a lot of power to use, therefore the computer needs to be more powerful than the ones we have in 2020. But, if we go past that problem, we should have a conscience in a computer program. With all this brain mapping becoming a reality, we have to consider the future impacts. The answer to our question from the proposal is all the parts of our brain working together is how conscience arises, that deduction is a big step in finding the truth. We can understand where consciousness comes from now. The impacts of using consciousness and AI to its full potential are limitless, and will change humanity for the better (Mashour, 2013).

\section{Future Impacts}

When people think of these subjects, it boggles their minds until they realize that we need to learn more about consciousness for the sake of our species. If we don't even know ourselves, how can we progress? There are several reasons as to why we need to do more research on this subject, and the uses that it can reveal. First off, being able to use true AI to do work only humans could once do is great. For example, if there are random objects needing sorting, you don't have to waste time doing it, but just let a 
robot work it out by itself. Also, in the future, we can upload all the learning skills to our brains, we can advance and learn extremely fast. If possible, the understanding of consciousness can make us find out things like how to hack our brains and do things we couldn't do before, or we can even upload our own conscience onto a computer into an android. If we get to a point where we can upload our own conscience into robots, then humans have technically won the battle of species. Robots can't get tired, therefore we can produce energy more efficiently. We just basically stopped diseases and almost anything organic that can harm us. The one thing that can happen if we upload ourselves that may seem very sci-fi is a virus that overtakes us. The probability is actually very high, since we are all electronic in this theoretical future. It is possible for one human to create a virus to spread and "shut down" everyone.

Since we pinpointed that consciousness is directly associated with our brains, we can try matching it to our definition. We match perfectly with Thomas Nagel's definition, we feel therefore we are aware, after the proposal, a few extra questions and ideas on AI, we understand our brain a lot more now. With a better understanding of our brains, we can eradicate many or most diseases and cancers involving the brain. By knowing more about our cerebrals, we can also modify our cerebrum to think faster and more efficiently. We can essentially hack our encephalon, since we know every nook and cranny of it and make humans an extremely advanced civilization. All these things are extremely far into the future, but if we never even attempt to unravel the mysteries of our brain then we may as well never progress or dream at all (Kumar, 2019).

To go back to tasks we can achieve more easily with the help of researched consciousness and conscious AI, we can use robots to help us think of the global temperature crisis' solutions and carry out those tasks. They are a higher level of life forms compared to us, but we should always have something to counteract them just in case they go rogue. We don't need to waste human energy, but since robots don't feel pain, they can help us do the work that old robots without conscience once couldn't. They could help us plan and build. Instead of just carrying out our ideas, they can make their own. They may even come up with newer and better solutions for our world. Robots don't have to function off sleep or thirst, so they don't take up many resources and are more efficient. Just like fire, this step is very crucial in helping our species to advance. By making this, it helps all our other projects, and will allow us to grow and build faster than before (Mashour, 2013).

To continue, I want to also go into detail about issues that consciousness can help with. For example, space exploration can be more advanced, because if our conscience can be transferred, then technically we could travel forever through space to discover. That also depends if our consciousness ages. In a book series called The Fifth Wave (Yancey, 2013), there are aliens that are pure consciousness, and they have travelled through space for millennia. Now that might actually be possible with humans. If we are going to do this, we should start with animals that have a smaller brain that we can replicate, then try and transfer or recreate their brain. Before our intelligence and awareness, we couldn't fly nor run very fast, but we developed intelligence, conscience and even speech. It was a big step when we became aware of our surroundings and noticed we could grow food instead of chasing it. So now we should be able to travel in space to solve our problems on earth, like overpopulation (Shekhtman, 2019).

Our cerebrum is responsible for sending out chemicals that strengthen our bodies. Adrenaline is controlled by the brain, and if we can sort of exploit that chemical and use it to our advantage, then humans would work more efficiently. Learning more on our brain and consciousness can help us understand how we can possibly live forever by transferring our cerebrum to a program or learning how to exploit our complex brain to become stronger, faster and overall a species that progresses (Collins, 2017 ). 
Right now, we are safe from robots taking over every job, but once they are conscious, I assume we will all either have to have no jobs or create new ones. We essentially just created a new super powered species that are indestructible. But if we can control them, then we can use them to help us advance with other things, like finding out how people can slow down the death of their cells. If we can use a robot to study and create ideas like a human but faster, we would become immortal. If we can put a human brain on a microchip, we have all the time in the universe to do whatever we want. But our consciousness has been described. We've pinpointed its roots, and understand how our brain has to work together to create, hold and initiate ideas and memories.

\section{Answers and Results}

From everything I just explained and theorized the answer to my question of the possibility of AI consciousness is most likely. Now I say this because technically we can't know the future for sure, but under the circumstances and technological advancements, I can guarantee that humans will come to a point where we can map the human brain. Many universities have already started the process of mapping and creating AI and artificial brains. The main barrier that we need to overcome is the mystery of the human brain.

But the main question of what is consciousness is what I need to answer. The answer is many things. We use the perspectives from different areas to create a definition that most professionals would agree upon, which is Thomas Nagel's definition "to feel or need something that you are not programmed to do by another organism." Basically, having awareness is knowing you exist in a world, and having some freewill and logic of some sorts. Then, by matching the definition with AI, I pinpointed the location of consciousness to the brain. From there, I talked about brain scans and how our consciousness is an ecosystem that works together to keep its host (you) alive. This trait we call consciousness is a super weapon in species, and we concluded what it is and its definition. By understanding where it comes from and what it is, this can create a big impact in our future.

In the future, I would like to dive deeper into what allowed humans to gain consciousness. What was the trait that allowed us humans to develop such large and complex brains? It could've been nature, or there was somehow an extremely rare change down our ancestry which changed our lives. I still have many questions about all the information that I've gathered. I know that people are discovering new things every day and maybe someday we can unlock the secret of our brain. But as you can see, one question unlocks many others, like from consciousness down to the path of AI.

I truly think that we need to understand the subject of consciousness more because of how much it can change our lives. It may have the same impact as when we (as a species) first learned how to control fire. We may think it's a waste of time, but a while back humans thought why they should waste their time on learning agriculture when they need to go hunt and gather. Some things don't seem important until it's done, and I think that this subject is one of them. Exploring a new area can also lead to solutions on earth like our overpopulation or climate control. We can cure diseases, save our planet, be more energy efficient, and maybe even hack our brains. We can genetically modify our cerebrals so humans can do things faster and more efficient. If we can discover everything about our brains and somehow map it then we have essentially defeated everything. When our brains become immortal, we have won the war that we have been secretly fighting; the war of time. We don't need to rush anymore, because we have all the time we really need to discover and invent. Understanding this one thing is the hack to standing over everything. 


\section{References:}

Boldrini, M. (2018). Older adults grow just as many new brain cells as young people. ScienceDaily. Retrieved from www.sciencedaily.com/releases/2018/04/180405223413.htm

Cherry, K. (2019). Comparing the Amount of Neurons in Human and Different Animal Brains. Retrieved from https://www.verywellmind.com/how-many-neurons-are-in-the-brain-2794889

Collins, R. (2017). Exercise, Depression, and the Brain. Retrieved from https://www.healthline.com/health/depression/exercise\#1

DePung, M. (2017). What Is Spiritual Consciousness, Awakening?: It's Not That Complicated - to Start! Retrieved from https://medium.com/@michaeldepung/what-is-spiritual-consciousnessawakening-its-not-that-complicated-to-start-c35aaa3211d2

Dror, N. (2015). When Exponential Progress Becomes Reality. Retrieved from https://medium.com/@nivo0o0/when-exponential-technological-progress-becomes-our-reality74acafd65e26

Falk, D., \& Quanta Magazine. (2019). How Artificial Intelligence Is Changing Science. Retrieved from https://www.quantamagazine.org/how-artificial-intelligence-is-changing-science-20190311/

Glasgow, R. (2018). Minimal Selfhood and the Origins of Consciousness. Retrieved from https://opus.bibliothek.uni-wuerzburg.de/frontdoor/index/index/docId/15747

Graziano, M. (2016). A New Theory Explains How Consciousness Evolved. Retrieved from https://www.theatlantic.com/science/archive/2016/06/how-consciousness-evolved/485558/

Harris, A. (2019). Conscious: a brief guide to the fundamental mystery of the mind. S.1.: HarpersPerennial.

Hines, T. (2018). Brain Anatomy, Anatomy of the Human Brain. Retrieved from https://mayfieldclinic.com/pe-anatbrain.htm

Keefe, J. (2014). The Nobel Prize in Physiology or Medicine 2014. Retrieved from https://www.nobelprize.org/prizes/medicine/2014/press-release/

Kumar, S. (2019). Advantages and Disadvantages of Artificial Intelligence. Retrieved from https://towardsdatascience.com/advantages-and-disadvantages-of-artificial-intelligence182a5ef6588c

MacDonald, F. (2018). Harvard Scientists Think They've Pinpointed The Physical Source of Consciousness. Retrieved from https://www.sciencealert.com/harvard-scientists-think-they-vepinpointed-the-neural-source-of-consciousness

Marlow, K. (2013). What is Consciousness? Retrieved from https://www.psychologytoday.com/ca/blog/the-superhuman-mind/201303/what-is-consciousness

Mashour, G. A., \& Alkire, M. T. (2013). Evolution of consciousness: Phylogeny, ontogeny, and emergence from general anesthesia. Retrieved from https://www.pnas.org/content/110/Supplement_2/10357\#ref-52

Shekhtman, S. (2019). NASA Applying AI Technologies to Problems in Space Science. Retrieved from https://www.nasa.gov/feature/goddard/2019/nasa-takes-a-cue-from-silicon-valley-to-hatchartificial-intelligence-technologies/ 